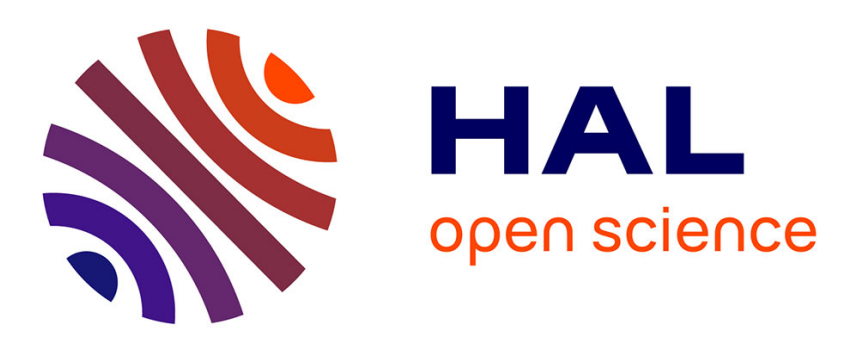

\title{
Angle-resolved magnetic and transport properties of Pr0.7Sr0.3MnO3 thin films
}

\author{
W. Prellier, Ch. Simon, J. Wolfman, B. Mercey
}

\section{To cite this version:}

W. Prellier, Ch. Simon, J. Wolfman, B. Mercey. Angle-resolved magnetic and transport properties of Pr0.7Sr0.3MnO3 thin films. Journal of Applied Physics, 1998, 83 (11), pp.7186-7188. $10.1063 / 1.367860$. hal-02176518

\section{HAL Id: hal-02176518 \\ https://hal.science/hal-02176518}

Submitted on 8 Jul 2019

HAL is a multi-disciplinary open access archive for the deposit and dissemination of scientific research documents, whether they are published or not. The documents may come from teaching and research institutions in France or abroad, or from public or private research centers.
L'archive ouverte pluridisciplinaire HAL, est destinée au dépôt et à la diffusion de documents scientifiques de niveau recherche, publiés ou non, émanant des établissements d'enseignement et de recherche français ou étrangers, des laboratoires publics ou privés. 


\title{
Angle-resolved magnetic and transport properties of $\mathrm{Pr}_{0.7} \mathrm{Sr}_{0.3} \mathrm{MnO}_{3}$ thin films
}

\author{
J. Wolfman, ${ }^{\text {a) }}$ W. Prellier, Ch. Simon, and B. Mercey \\ Laboratoire Crismat, UMR CNRS 6508, ISMRA, Bd du $M^{a l}$ Juin 14050, Caen cedex, France
}

In this communication, the variation of the resistance and of the magnetization with respect to the angle between the applied magnetic field and the substrate normal is discussed for a $\operatorname{Pr}_{0.7} \mathrm{Sr}_{0.3} \mathrm{MnO}_{3}$ thin film at $30 \mathrm{~K}$. An angular hysteresis is observed for these properties and this behavior is correlated with the magnetic domain wall motion. It is shown that the number of domain walls is controlled through the rotation of the applied magnetic field, and that these domain walls play an important part in the colossal magnetoresistance behavior of these films. Furthermore, a model describing the reversal of the magnetization with the rotation of the magnetic field has been developed. This model enables one to extract the dependence of the resistance upon the direction of the magnetization with respect to the crystalline axis. (C) 1998 American Institute of Physics. [S0021-8979(98)52511-X]

Manganese perovskites exhibiting colossal magnetoresistance (CMR) have drawn large interest these last few years, particularly for the realization of thin films with technological applicability. ${ }^{1,2}$ However, the microscopic mechanism of CMR is not clearly established. The double exchange (DE) interaction, ${ }^{3}$ which has been classically involved to explain CMR, relates the hopping rate of an $e_{g}$ hole between two Mn sites to the angle between the respective moments of these Mn sites. Yet this interaction does not consider the direction of the moments with respect to the hopping direction (i.e., the local current direction) nor to the crystalline direction. Eckstein et al. have shown ${ }^{4}$ that the resistivity of a $\mathrm{La}_{0.66} \mathrm{Ca}_{0.33} \mathrm{MnO}_{3}$ thin film depends upon the angle between the in-plane applied magnetic field and the current direction. They have compared this effect to the anomalous magnetoresistance (AMR) observed in metal thin films. However, this resistivity variation could also be related to the direction of the Mn moments with respect to the crystalline axis. In order to investigate this possibility, we have carried out magnetic and transport measurements on a $\mathrm{Pr}_{0.7} \mathrm{Sr}_{0.3} \mathrm{MnO}_{3}$ thin film, while varying the direction of the applied magnetic field, $\mathbf{B}$, with respect to the crystalline axis, and keeping $\mathbf{B}$ perpendicular to the current direction.

In situ annealed $\mathrm{Pr}_{0.7} \mathrm{Sr}_{0.3} \mathrm{MnO}_{3}$ thin films were grown on $\mathrm{LaAlO}_{3}$ substrates by pulsed laser deposition. The microstructure has been investigated by $\mathrm{x}$-ray diffraction and transmission electron microscopy (TEM), and is discussed, with respect to the growth conditions, in details elsewhere. ${ }^{5}$ TEM has evidenced that the films crystallize epitaxially and with the space group Pbnm. The growth axis is in the [110] direction $\left(a \approx b \approx \sqrt{2} a_{p}\right)$ and the $\mathbf{c}$ axis is in the plane of the film $\left(c \approx 2 a_{p}\right)$. The pseudocubic structure of this material, identical to that obtained by rf sputtering, ${ }^{6}$ gives rise to the coexistence of domains with perpendicular $\mathbf{c}$ axis. The thickness of the films was determined to be $7000 \AA$ (mechanical step measurement).

a) Author to whom correspondence should be addressed; electronic mail: wolfman@crismat.ismra.fr
Electrical measurements, registered by the four-probe method along the [100] direction of $\mathrm{LaAlO}_{3}$, evidence a transition from an activated state $\left(E_{a}=108 \mathrm{meV}\right)$ to a metallic state at $T=240 \mathrm{~K}$ as the temperature is decreased. This transition is correlated with the onset of ferromagnetic ordering at $T_{C}=252 \mathrm{~K}$. These temperatures are comparable to those observed in bulk materials $\left(T_{c}=259 \mathrm{~K}\right){ }^{7}$ A magnetic field of $70 \mathrm{kG}$, applied in the plane of the film and perpendicular to the current $I$, gives rise to a CMR $\left(R_{0} / R_{70 \mathrm{kG}}\right)$ of $400 \%$ at $T=200 \mathrm{~K}$. The spontaneous magnetization of this composition has been reported to be $564 \mathrm{emu} / \mathrm{cm}^{3}$ at $5 \mathrm{~K}$ (neutron diffraction ${ }^{7}$ ), i.e., locally, the magnetic moments are almost completely polarized. The maximum magnetization measured was $426 \mathrm{emu} / \mathrm{cm}^{3}$ in a magnetic field of $17 \mathrm{kG}$ at $5 \mathrm{~K}$, evidencing the existence of magnetic domains.

Magnetic and transport measurements presented below, were made at a constant field modulus $B$ and at $30 \mathrm{~K}$. The direction of $\mathbf{B}$ was varied inside a plane containing the substrate [010] direction and the substrate normal $(\mathbf{B} \perp \mathbf{I}) . \Theta_{B}$, defined as the angle between the $\mathbf{B}$ direction and the substrate normal, was varied from $-90^{\circ}$ to $+90^{\circ}$. Furthermore, transport measurements were made as $\Theta_{B}$ was varied back to $-90^{\circ}$.

The $\Theta_{B}$ dependence of the resistance and of the magnetization are given in Figs. 1 and 2, respectively, and will be discussed together. It should be noted that the measured magnetic moment, herein called $M \|$ (see Fig. 2), is the component of the global moment $\mathbf{M}$ along the $\mathbf{B}$ direction. Thus, if $\Theta_{M}$ is defined as the angle between $\mathbf{M}$ and the substrate normal, $M \|=M \cos \left(\Theta_{M}-\Theta_{B}\right)$. The observed decrease of $M \|$ at low fields [Fig. 2(b)] results from two effects. First, as M rotates out of the plane of the film, the internal field $H_{i}$ decreases due to the increase of the demagnetizing field $\mathbf{H}_{d}$ $\left[H_{d}=4 \pi M \cos \left(\Theta_{M}\right)\right]$, leading to a decrease of $M$. Second, as $\mathbf{B}$ rotates out of the plane, $\mathbf{M}$ tends to follow $\mathbf{B}$, but is delayed with respect to $\mathbf{B}$ owing to $\mathbf{H}_{d}$, increasing $\Theta_{M}$ $-\Theta_{B}$. At $600 \mathrm{G}$ [Fig. 1(a)], the resistance presents a large hysteresis and two maximum. For $\mathbf{B}=2500 \mathrm{G}$ the hysteresis tends to be restricted around $\Theta_{B}=0^{\circ}$. For a further $B$ in- 


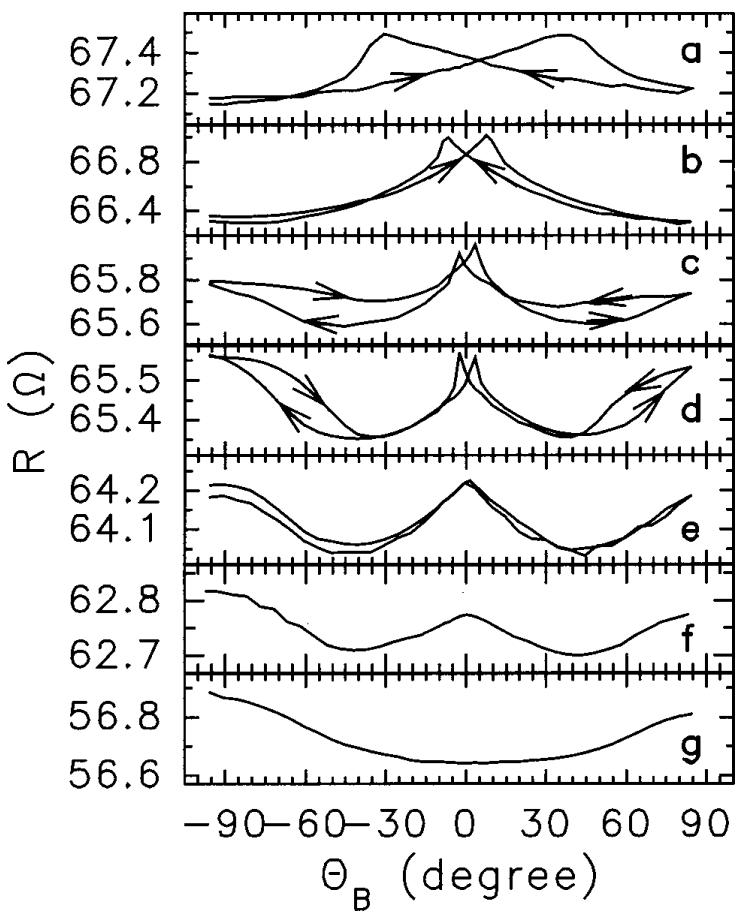

FIG. 1. $\Theta_{B}$ dependence of the resistance in an applied magnetic field equal to (a) $600 \mathrm{G}$, (b) $2.5 \mathrm{kG}$, (c) $4.5 \mathrm{kG}$, (d) $5.5 \mathrm{kG}$, (e) $12.5 \mathrm{kG}$, (f) $20 \mathrm{kG}$, and (g) $60 \mathrm{kG}$.

crease [Figs. 1(c) and 1(d)], a hysteresis develops for higher $\Theta_{B}$, the resistance maximum moving toward $\Theta_{B}=0$. The maximum of resistance are expected to arise as $\mathbf{M}$ is perpendicular to the substrate (maximum $\mathbf{H}_{d}$ ). The fact that they do not appear for $\Theta_{B}=0$ denotes that $\mathbf{M}$ is delayed with respect to $\mathbf{B}$. A decrease of the resistance is also observed for $\mathbf{B}$ $\geqslant 4500 \mathrm{G}$ around $\Theta_{B}=45^{\circ}$ and $0^{\circ}$. For $\mathbf{B}>7000 \mathrm{G}$, the resistance is reversible with respect to $\Theta_{B}$. The reversibility (or the irreversibility) of $R\left(\Theta_{B}\right)$ upon $\mathbf{B}$ can be accounted for by the magnetic domain wall motion. The $M(B)$ curve at 30 $\mathrm{K}$ with $\mathbf{B} \|$ to the film (not shown) indeed presents hysteresis of the magnetization for $\mathbf{B}<3500 \mathrm{G}$. This hysteresis, due to domain wall motion, is thus reversible (irreversible) for $\mathbf{B}$ $>3500 \mathrm{G}(\mathbf{B}<3500 \mathrm{G})$. In the out-of-plane magnetic field

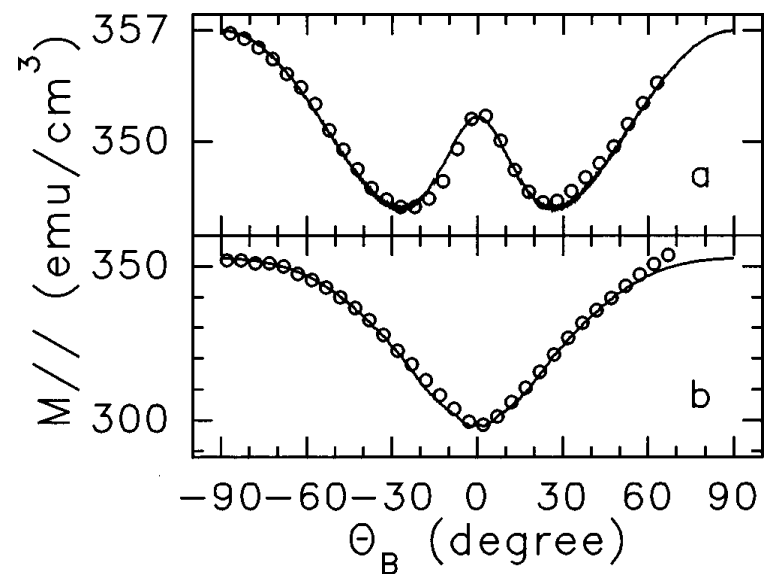

FIG. 2. $\Theta_{B}$ dependence of $M \|$ (see text) for $B=20 \mathrm{kG}$ (a) and $B=7.5 \mathrm{kG}$ (b) (circles) accompanied by the correspondent fits (solid lines) from the model (see text).

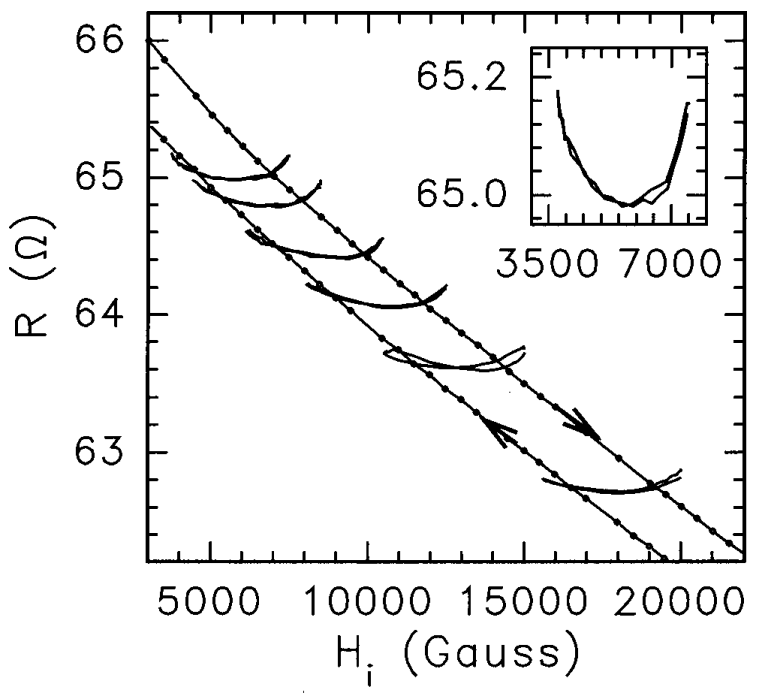

FIG. 3. $\mathbf{H}_{i}$ dependence of the resistance extracted from the $R\left(\Theta_{B}\right)$ curves (solid lines) in an applied $\mathbf{B}$ of (from bottom to top): $20 \mathrm{kG}, 15 \mathrm{kG}, 12.5 \mathrm{kG}$, $10.5 \mathrm{kG}, 8.5 \mathrm{kG}, 7.5 \mathrm{kG}$. In insert, $B=7.5 \mathrm{kG}$. Also shown in $R(H)$ while $H$ is ramped from $0 \mathrm{G}$ to $70 \mathrm{kG}$ and back to $0 \mathrm{G}$ (lines with dots) in the plane of the film.

set up described above, $\mathbf{H}_{d}$ is about $4000 \mathrm{G}$ for $\mathbf{B}=7500 \mathrm{G}$ and $\Theta_{M}=0$ [see Fig. 2(b) for $\Theta_{B}=-90$ ], leading to an internal field of about $3500 \mathrm{G}$.

To establish the correlation between $\Theta_{M}$ and $\Theta_{B}$ at a fixed applied field, we have developed a model describing $\mathbf{M}\left(\Theta_{B}\right)$, which takes into account the applied field $\mathbf{B}$, the demagnetizing field $\mathbf{H}_{d}$, and the magnetocrystalline energy. The magnetization was assumed to be homogeneous to define $\mathbf{H}_{d}$. Furthermore, we assumed that $\mathbf{M}$ is pointing in the direction of the internal field $\mathbf{H}_{i}\left(\mathbf{H}_{i}=\mathbf{B}+\mathbf{H}_{d}\right)$. The total energy is minimized in a step-by-step calculation, varying $\Theta_{B}$. This model does not account for hysteresic behavior, and was then used for $B>7000 \mathrm{G}$. A detailed description of the model will be published elsewhere. The calculations result in a good quantitative agreement as exemplified by that displayed in Fig. 2 (solid lines). Thus, the variation of $\Theta_{M}$ and $\mathbf{H}_{i}$ as functions of $\Theta_{B}$ can be calculated by this model.

We have represented the variation of the resistance extracted from the $R\left(\Theta_{B}\right)$ curves as a function of $\mathbf{H}_{i}$ in Fig. 3 (the minimum $\mathbf{H}_{i}$ is for $\Theta_{B}=0$ and corresponds to the maximum $\mathbf{H}_{d}$ ). Figure 3 also displays the variation of the resistance upon a magnetic field $\mathbf{H}[R(H)]$, while the latter is applied along the [010] direction of the substrate (i.e., $\mathbf{H}_{i}$ $=\mathbf{H}$ ), and ramped from 0 to $70 \mathrm{kG}$ and back to $0 \mathrm{G}$. It appears that the resistance extracted from the $R\left(\Theta_{B}\right)$ curves are much less sensitive to $\mathbf{H}_{i}$ than is $R(H) . R(H)$ is hysteretic, resulting from a different magnetic state before and after the application of a $70 \mathrm{kG}$ field: the number of magnetic domains can be assumed to be different, giving rise to an increase (decrease) of the scattering rate of the carriers as the number of domain walls increases (decreases). The $R\left(\mathbf{H}_{i}\right)$ curves deduced from the $R\left(\Theta_{B}\right)$ curves are reversible and join the two branches of the $R(H)$ curve as $\mathbf{H}_{i}$ varies. This indicates a nucleation of magnetic domains as $\mathbf{M}$ rotates from $\Theta_{M}=0$ to $\Theta_{M}=90$. The dependence of the resistance upon $\mathbf{H}_{i}$ (see the inset of Fig. 3) results from the onset of two 
antagonist effects. As $\mathbf{M}$ rotates from $\Theta_{M}=90$ to $\Theta_{M}=0, \mathbf{H}_{i}$ decreases, giving rise to a slight decrease of $\mathbf{M}$. From the DE point of view, decreasing $\mathbf{M}$ leads to a decrease of the hopping rate, i.e., an increase of the resistance. However, it has been shown that, during the same rotation $\left(\Theta_{M}=90-\Theta_{M}\right.$ $=0$ ), the number of domain walls decreases, tending to decrease the resistance. The competition between the decrease of both $\mathbf{M}$ and the number of domain walls explains the not monotonous variation of $R$ upon $\mathbf{H}_{i}$. The $R\left(\Theta_{B}= \pm 90\right)$ values are systematically higher than the $R(H)$ values from the upper branch (field increasing) and can be accounted for by the existence of a larger number of magnetic domain walls, since the $R\left(\Theta_{B}\right)$ curves were registered one after the other, increasing $\mathbf{B}$. Since $R$ is not monotonous in $\mathbf{H}_{i}$, it appears that $\mathbf{H}_{i}$ is not the primary factor in determining $R\left(\Theta_{B}\right)$.

To investigate the $\Theta_{M}$ dependence of the resistance, we have plotted in Fig. 4 the normalized $R\left(\Theta_{M}\right)$ curves for four different $\mathbf{B}$. These curves are field independent for $\Theta_{M}$ $\geqslant 60^{\circ}$ and diverge for lower $\Theta_{M}$ values. Because of the coexistence of magnetic domains, there is some deviation of the local magnetic moment direction $\left(\Theta_{m}\right)$ with respect to the global moment direction $\left(\Theta_{M}\right) ; \Theta_{M}$ is then the spatial average value of the $\Theta_{m}$. In a plot displaying the total energy as a function of $\Theta_{M}$ for a fixed $\Theta_{B}$, there appears two local minima with a difference energy $\Delta E$. The probability of a local moment direction to shift from one local minimum to the other is related to $\Delta E$ : the lower $\Delta E$ is, the more easily $\Theta_{m}$ shifts ( $\Delta E$ has to be compared to the energy cost $E_{n}$ of a domain nucleation). The calculation shows that as $\Theta_{B}$ decreases, $\Delta E$ decreases and minimizes for $\Theta_{B}=0$. Local magnetic direction fluctuations are then favored by a decrease of $\Theta_{B}$ (which implies a decrease of $\Theta_{M}$ ). For a fixed $\Theta_{B}, \Delta E$ depends also upon $\mathbf{B}$ : the higher $\mathbf{B}$ is, the higher $\Delta E$ is. In the field independent part of the $R\left(\Theta_{M}\right)$ curves, the deviations of $\Theta_{m}$ around $\Theta_{M}$ are negligible, which implies that $\Delta E$ is large compared to $E_{n}$. For $\Theta_{B}<60^{\circ}$, the $R\left(\Theta_{M}\right)$ curves separate, and their different shapes are thus due to different $\Delta E$ (for a fixed $\Theta_{B}$ and $\Theta_{M}$ ) owing to the different applied magnetic fields. If we assume that deviations of $\Theta_{m}$ around $\Theta_{M}$ are negligible for $\mathbf{B}=60 \mathrm{kG}$ (i.e., $\Theta_{M} \cong \Theta_{m}$ $\cong \Theta_{B}$ ), we can describe the real dependence of the resistance

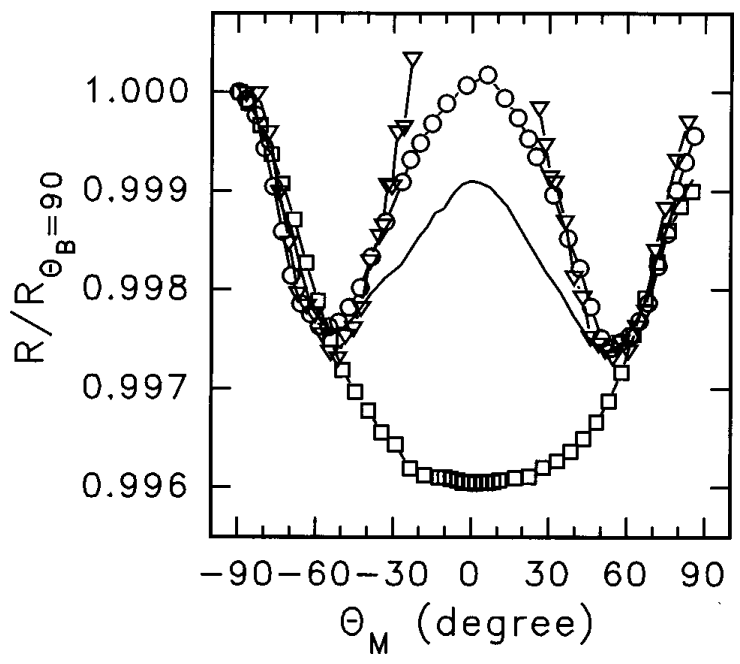

FIG. 4. $\Theta_{M}$ dependence of the resistance extracted from the $R\left(\Theta_{B}\right)$ curves, normalized to $R\left(\Theta_{B}=-90^{\circ}\right)$, in an applied $\mathbf{B}$ of: $7.5 \mathrm{kG}$ (triangles), 12.5 $\mathrm{kG}$ (circles), $20 \mathrm{kG}$ (solid line) and $60 \mathrm{kG}$ (squares).

upon $\mathbf{M}$ as follows: the resistance is maximum when $\mathbf{M}$ is in the film plane and is minimum when $\mathbf{M}$ is perpendicular to the film. At lower fields, the fluctuations of $\Theta_{m}$ around $\Theta_{M}$ imply the creation of magnetic domain walls which take part in the observed increase of the resistance around $\Theta_{B}=0$ (inset of Fig. 3). Finally, it has been shown that AMR is not the only anisotropic effect involved in CMR thin films; the resistance also depends upon the direction of the magnetization with respect to the crystalline axis.

${ }^{1}$ R. M. Kusters, J. Singleton, D. A. Keon, R. M. Greedy, and W. Hayes, Physica B 155, 362 (1989).

${ }^{2}$ S. Jin, H. M. O'Bryan, T. H. Tiefel, M. Mc Cormack, and W. W. Rhodes, Appl. Phys. Lett. 66, 382 (1995).

${ }^{3}$ P. D. De Gennes, Phys. Rev. 118, 141 (1960).

${ }^{4}$ I. Bozovic, J. N. Eckstein, J. O’Donnell, M. Onellion, and M. S. Rzchowski, Appl. Phys. Lett. 69, 9 (1996).

${ }^{5}$ J. Wolfman, W. Prellier, B. Mercey, and Ch. Simon (unpublished).

${ }^{6}$ B. Mercey, Ph. Lecoeur, M. Hervieu, J. Wolfman, Ch. Simon, H. Murray, and B. Raveau, Chem. Mater. 9, 1177 (1997).

${ }^{7}$ K. Knirzeck, Z. Jirak, E. Pollert, and F. Zounova, J. Solid State Chem. 100, 292 (1992). 